\title{
High throughput sequencing of two celery varieties small RNAs identifies microRNAs involved in temperature stress response
}

\author{
Meng-Yao Li, Feng Wang, Zhi-Sheng Xu, Qian Jiang, Jing Ma, Guo-Fei Tan and Ai-Sheng Xiong*
}

\begin{abstract}
Background: MicroRNAs (miRNAs) are small, non-coding RNAs of 20 to 24 nucleotides that regulate gene expression and responses to biotic and abiotic stress. Till now, no reports have previously been published concerning miRNAs in celery.

Results: Two small RNAs libraries were constructed from two celery varieties, 'Jinnan Shiqin' and Ventura', and characterized by deep sequencing. A total of 431 (418 known and 13 novel) and 346 (341 known and five novel) miRNAs were identified in celery varieties 'Jinnan Shiqin' and 'Ventura', respectively. Potential miRNA-target genes were predicted and annotated by screening diverse protein databases, including Gene Ontology, Cluster of Orthologous Groups and Kyoto Encyclopedia of Genes and Genomes. Significant differential expression between the two varieties was seen for 221 miRNAs. qRT-PCR was used to analyze the abundance of six miRNAs under cold and heat stress conditions. The results showed that miRNAs may have important functions in controlling temperature stress in celery.

Conclusion: A large number of miRNAs were identified in celery, and their target genes, functional annotations, and gene expression patterns have been explored.

These findings provide the first information on celery miRNAs and enhance understanding of celery miRNA regulatory mechanisms under extreme temperature stress.
\end{abstract}

Keywords: microRNAs, Temperature stress, Deep sequencing, qRT-PCR, Celery

\section{Background}

MicroRNAs (miRNAs) are small, non-coding RNAs found in animals and plants, and mainly function in regulating gene expression at the post-transcriptional level [1]. miRNAs are highly conserved in eukaryotes, and are an important component in the evolution of genetic regulation [2,3]. Mature miRNAs are generated from primary miRNAs (pri-miRNAs) via two steps. The pri-miRNAs are cut by RNA polymerase II into precursor miRNAs (pre-miRNAs) that have a stem-loop structure and are 70 to 100 nucleotides (nt) in length. The pre-miRNAs are then cleaved into mature miRNAs of 19 to $23 \mathrm{nt}$ by Dicer-Like1 in the cytoplasm [4].

The first two characterized miRNAs, lin-4 and let-7, were identified as regulators of the juvenile-to-adult phase

\footnotetext{
* Correspondence: Xiongaisheng@njau.edu.cn

State Key Laboratory of Crop Genetics and Germplasm Enhancement, Ministry of Agriculture Key Laboratory of Biology and Germplasm Enhancement of Horticultural Crops in East China; College of Horticulture, Nanjing Agricultural University, Nanjing, People's Republic China
}

transition in Caenorhabditis elegans [5,6]. The latest miRBase database (miRBase19) contains 21,264 entries representing hairpin pre-miRNAs, expressing 25,141 mature miRNA products in 193 species. Numerous studies have revealed that miRNAs are involved in diverse biological and metabolic processes, such as regulation of cell growth and development, abiotic stress response, pathogen defense and gene translational repression [7-10]. Levels of miR156, miR167, and miR164 increase during virus intrusion $[11,12]$. Furthermore, miR172 and miR159 affect flowering time regulation in plants $[13,14]$, whereas miR160 has important roles in the regulation of plant development and hormonal signals [15]. In cold conditions, miR393 overexpression is induced [16].

Celery (Apium graveolens L.) is an annual or perennial herb belonging to the Apiaceae family. Although celery originated from the Mediterranean and the Middle East, it is now cultivated worldwide. Celery is rich in carotene, vitamins, carbohydrates, and volatile aroma compounds, and has excellent medicinal properties to regulate the digestive 
system and blood lipids [17-19]. However, celery is one of the most common allergenic foods in many European countries [20]. Numerous allergenic compounds contained in celery tissue can induce allergic symptoms [21]. miRNAs have significant biological functions in metabolic and immune responses [8,22], and exploring the miRNAs present in celery will provide a foundation for the study of their functions. Till now, there have been no previously published reports concerning miRNA s in Apiaceae species.

Extreme temperature is one of the major limiting factors on celery growth and yield. Low temperatures can lead to early bolting, and high temperatures can induce various diseases. To investigate the roles of celery miRNAs under temperature stress, two varieties, 'Jinnan Shiqin' and 'Ventura', were selected for small RNA and transcriptome sequencing. 'Jinnan Shiqin' was bred by the Institute of Tianjin City, China, while 'Ventura' originated in the United States and was introduced to China. These two varieties have similar phenotypes, although they originated from different geographical areas. Here, the significant differential expression miRNAs were detected between two varieties, and the results provide useful information for miRNA that can response to temperature stress.

\section{Results}

\section{Sequence analysis of small RNAs}

To identify the low-abundance candidate miRNAs in the 'Jinnan Shiqin' and 'Ventura' celery varieties, Solexa sequencing was performed on the small RNAs libraries. A total of 6,951,840 and 6,057,911 reads were generated from 'Jinnan Shiqin' and 'Ventura' libraries, respectively. After filtering the reads for those of low quality, 3 ' insert null, poly(A), length $<16$ or length $>30$, the majority of small RNAs were 21 to $24 \mathrm{nt}$ in length (Figure 1). The length distributions of small RNAs were similar for both varieties. The 24 nt small RNAs were the most abundant, representing $35.75 \%$ and $24.50 \%$ of small RNAs in 'Jinnan Shiqin' and 'Ventura', respectively. This result is consistent with reports for other plants, such as Arabidopsis thaliana, trifoliate oranges, peanuts and olives [23-26]. Some studies have reported that $24 \mathrm{nt}$ siRNAs are involved in heterochromatin modification, particularly in genomes with high repetitive sequence content $[27,28]$.

The small RNAs were further classified into different categories by performing BLAST searches against Rfam. Noncoding RNAs included miRNA, rRNA, tRNA, snoRNA, snRNA, and other unannotated RNAs. More categories of small RNAs were present in 'Jinnan Shiqin' than in 'Ventura' (Table 1).

\section{Identification of conserved miRNAs}

To identify conserved miRNAs in the two celery varieties, small RNA sequences were compared with currently known mature plant miRNAs in the miRBase.

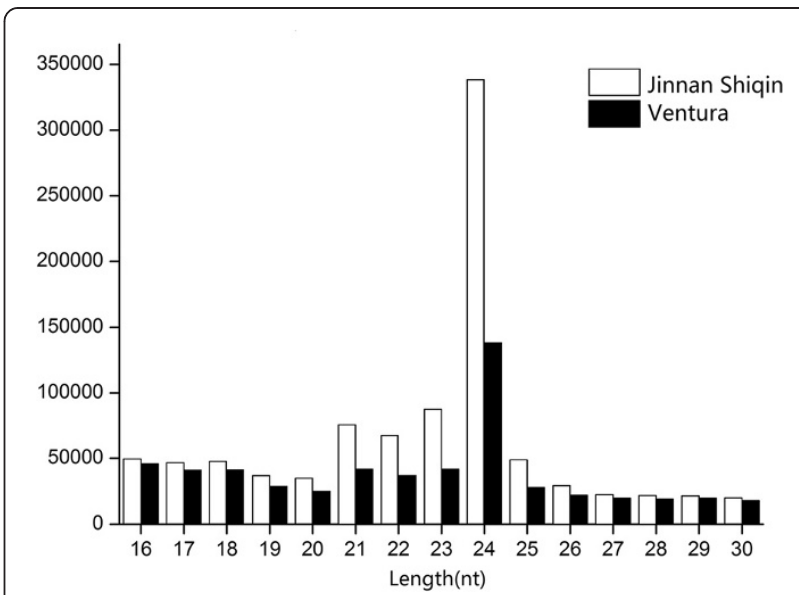

Figure 1 Length distributions of small RNAs in two celery varieties.

Total of 418 unique miRNA sequences belonging to 28 miRNA families were identified in 'Jinnan Shiqin', and total of 341 unique miRNA sequences belonging to 34 miRNA families were identified in 'Ventura' (Figure 2). Most of the identified miRNAs showed high sequence similarities to miRNAs in other plants. The distributions of miRNA families were similar between the two libraries. Several families, such as miR156, miR159, miR166, miR167-1 and miR396, were relatively abundant, whereas some families were not. The length of miRNAs and nucleotide preference distributions are shown in Figure 3. The majority of miRNAs tended to start with 5'-U and not with 5'-G, which is consistent with typical miRNA sequence patterns.

\section{Identification of novel miRNAs}

To identify novel miRNA sequences, all unannotated small RNAs were searched against transcriptome sequence data (SRX326597). After searching for potential pre-miRNAs and predicting their hairpin-like structures, 13 and five unique sequences were identified as novel miRNAs in 'Jinnan Shiqin' and 'Ventura', respectively (Table 2

Table 1 Distribution of small RNAs among different categories in 'Jinnan Shiqin' and 'Ventura'

\begin{tabular}{llllll}
\hline Category & \multicolumn{2}{l}{ 'Jinnan Shiqin' } & & 'Ventura' \\
\cline { 2 - 3 } & Unique tags & Total tags & & Unique tags & Total tags \\
\hline miRNA & 431 & 470923 & & 346 & 109348 \\
rRNA & 83264 & 786363 & 64745 & 898835 \\
tRNA & 17811 & 124663 & 13497 & 120933 \\
snoRNA & 12647 & 36493 & 11176 & 29581 \\
snRNA & 860 & 3121 & 801 & 2848 \\
Unannotated & 59714 & 212201 & 53110 & 197038 \\
Total & 1742714 & 1633764 & 143670 & 1358583 \\
\hline
\end{tabular}




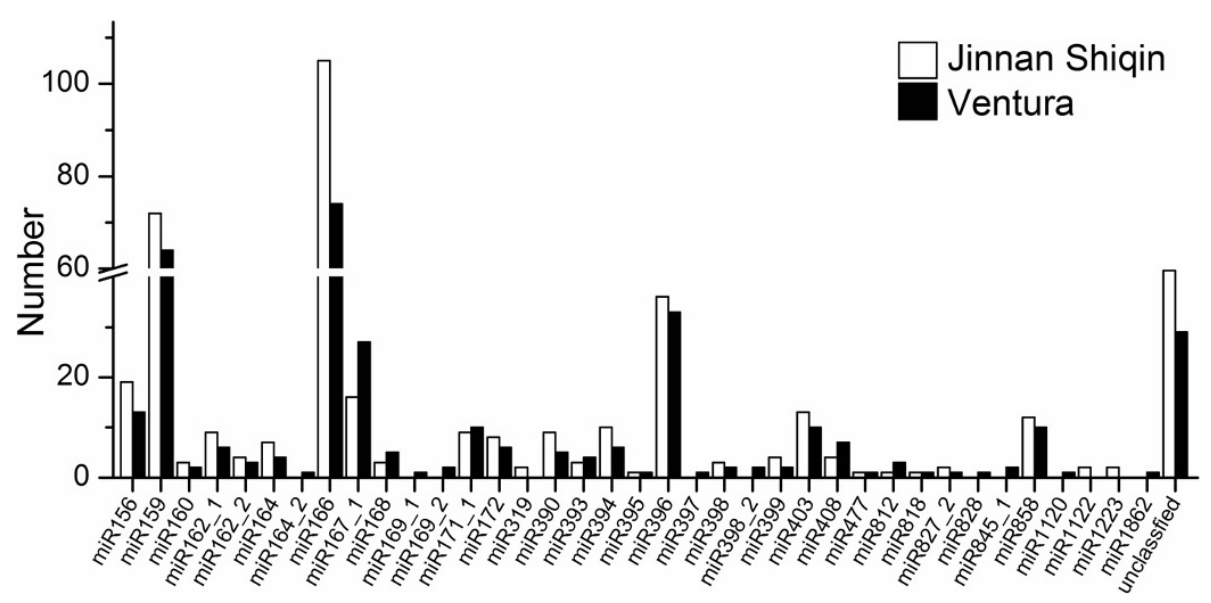

Figure 2 Numbers of identical miRNA members in each family in 'Jinnan Shiqin' and 'Ventura'.

and Table 3). Only one novel miRNA, celery-miR-27, was identified in both celery varieties. The novel miRNA sequences were 20 to $24 \mathrm{nt}$ in length, and $24 \mathrm{nt}$ reads were the most abundant. The range in length of pre-miRNAs was 77 to $102 \mathrm{nt}$ in 'Jinnan Shiqin', with an average of $94 \mathrm{nt}$. The average minimum free energy values in 'Jinnan Shiqin' and 'Ventura' were -23.18 and $-31.12 \mathrm{kcal} / \mathrm{mol}$, respectively, which are much higher than values for other plant premiRNAs $(-59.5$ and $-71.0 \mathrm{kcal} / \mathrm{mol}$ in Arabidopsis and rice, respectively) [29].

\section{Prediction and annotation of miRNA target genes}

To better understand the functions of the identified miRNAs, putative target genes were predicted using the psRNA Target program. A total of 503 and 408 potential target genes were identified in 'Jinnan Shiqin' and 'Ventura', respectively. To evaluate the potential functions of these miRNA target genes, GO analysis was used [30]. The miRNA target genes were categorized according to biological process, cellular component, and molecular function (Figure 4). Fifty-six genes were categorized as cellular components. Based on molecular function, 111 genes were classified into nine categories, among which the most over-represented GO terms were binding and catalytic activities. Eight different biological processes were found, among which the three most frequent terms were metabolic process, cellular process, and biological regulation. To further evaluate the completeness of the transcriptome and the effectiveness of the annotation process, the annotated sequences were searched for genes that could be assigned to Clusters of Orthologous Groups (COG) classifications [31]. Overall, 57 sequences were assigned to COG classifications (Figure 5), and COG-annotated putative proteins were functionally classified into at least 25 molecular families. The cluster for general function prediction was the largest group, followed in descending order by translation, secondary metabolite biosynthesis, and signal transduction. The biological interpretation of these target genes was further examined using KEGG pathway analysis. Twenty-six different pathways were found, and the most frequently represented pathways included those for transcription factors (ten enzymes), plant hormone signal transduction (four enzymes), chromosomes (four enzymes), and protein kinase inhibitors (3 enzymes).
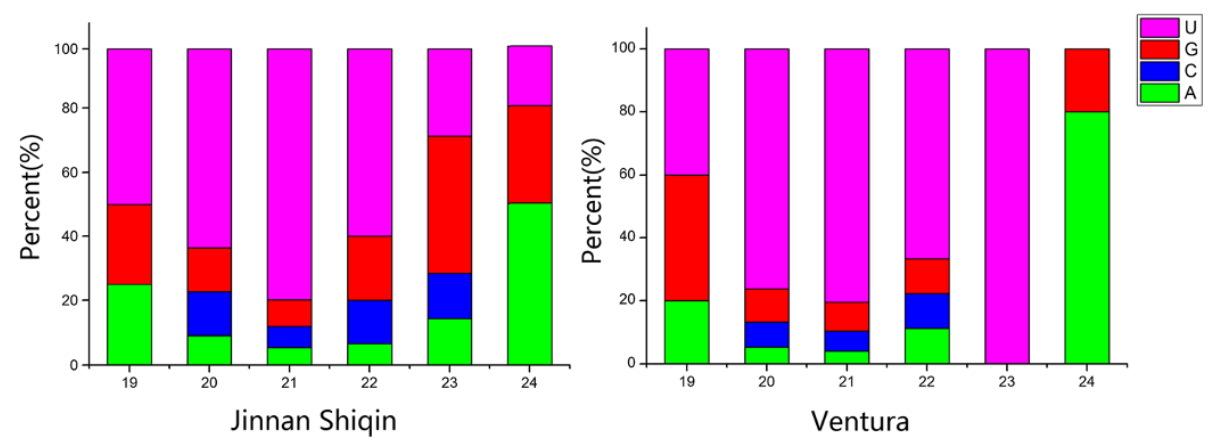

Figure 3 First nucleotide bias of miRNAs in 'Jinnan Shiqin' and 'Ventura'. 
Table 2 Novel miRNA candidates in 'Jinnan Shiqin'

\begin{tabular}{|c|c|c|c|c|c|c|}
\hline miRNA ID & Sequence $\left(5^{\prime}-3^{\prime}\right)$ & Length(nt) & Start/end precursor & Length of precursors(nt) & Arm & MFE (kcal/mol) \\
\hline celery-miR-1 & AAAATCGCTAGGCGGACCAGGGCG & 24 & $66-166$ & 101 & $3^{\prime}$ & -19.36 \\
\hline celery-miR-4 & ACAGGGGTCCATCACTATTAATAA & 23 & $535-629$ & 95 & $5^{\prime}$ & -19.43 \\
\hline celery-miR-5 & ACGAATTTAAAGATATTAACAATC & 22 & $263-363$ & 101 & $5^{\prime}$ & -18.90 \\
\hline celery-miR-7 & ACTGTAGTITTCATTGGTTITAC & 23 & 1903-1999 & 97 & $5^{\prime}$ & -20.40 \\
\hline celery-miR-9 & AGTGATTGATCTGTTTTGAT & 20 & 207-293 & 87 & $3^{\prime}$ & -23.90 \\
\hline celery-miR-14 & CGGAACCAGGATTITTGATGTCC & 23 & 93-194 & 102 & $5^{\prime}$ & -30.10 \\
\hline celery-miR-16 & GAACGGCGTCGGACGGAGCCGCCC & 24 & $499-585$ & 87 & $3^{\prime}$ & -26.40 \\
\hline celery-miR-17 & GCGGGCTGATCCGGGTTGAAGACA & 24 & $13-107$ & 95 & $3^{\prime}$ & -24.70 \\
\hline celery-miR-19 & GTTGTTATGTATTCGTTGTTATGA & 24 & $551-645$ & 95 & $3^{\prime}$ & -19.43 \\
\hline celery-miR-20 & GTITACTGCTAGAAGAAATGA & 22 & $3431-3507$ & 77 & $5^{\prime}$ & -21.00 \\
\hline celery-miR-21 & TAATGCTCTTCGTACTGCATC & 21 & 1089-1186 & 98 & $3^{\prime}$ & -33.50 \\
\hline celery-miR-24 & TCCGGATTCTCCTIIIITTCGAAC & 24 & 9-99 & 91 & $3^{\prime}$ & -19.30 \\
\hline celery-miR-27 & TTTAGCTTGAACTATAGATT & 20 & $1522-1617$ & 96 & $3^{\prime}$ & -24.90 \\
\hline
\end{tabular}

Differential expression of miRNAs between celery varieties The differences between the miRNA profiles for the two celery varieties are possibly related to differences in their responses to the surrounding environment. IDEG6 software was used to analyze the miRNAs of both varieties. A false discovery rate (FDR) $<0.001$ and an absolute threshold value of the $\log 2$ ratio fold-change $>1$ were used to determine the statistical significance of miRNA abundance. A total of 221 differentially expressed miRNAs were selected from the two libraries (Figure 6 and Additional file 1: Table S1). In 'Jinnan Shiqin', 55 genes were up-regulated, whereas 166 genes were down-regulated. Some families showed large differences in abundance between the two varieties. For example, mtr-miR166e and osa-miR396a were more abundant in 'Jinnan Shiqin', whereas sbi-miR166b and ghr-miR396b were more abundant in 'Ventura'.

qRT-PCR analysis of miRNAs following cold or heat stress In this study, six miRNAs (miR160, miR164, miR168, miR394, miR395 and miR408), whose expression was previously reported to be regulated by cold or heat stress, were analyzed after temperature treatment. The changed profiles of these miRNAs are shown in Figure 7. Almost all of these miRNAs were up-regulated by cold or heat stress in both celery varieties. Aside from miR168, the abundance of the miRNAs was higher under cold stress in 'Jinnan Shiqin' than in 'Ventura,' with the biggest change in miR164. After heat treatment, miR395 and miR408 were highly abundant in 'Jinnan Shiqin', whereas miR164, miR168, and miR394 were more abundant in 'Ventura'. These results indicate that these six miRNAs are involved in temperature stress responses during a relatively short period of time.

\section{Discussion}

High throughput sequencing technology has been extensively applied in small RNA research. Thousands of miRNAs and their functions have been identified in higher plants. To date, only a single report detailing transcriptome data in celery has been published, and thus, comprehensive miRNA information is not available for this plant [32]. Moreover, there are no reports concerning miRNAs in other species of Apiaceae. In the present study, miRNAs were identified and characterized from two celery varieties, namely, 'Jinnan Shiqin' and 'Ventura', which come from different geographical sources but have similar morphology. Till now, this study is the first to identify and investigate small RNAs in celery, and the results provide new information for further research into the functions, biological pathways and evolution of target genes related to temperature stresses in celery.

Table 3 Novel miRNA candidates in 'Ventura'

\begin{tabular}{|c|c|c|c|c|c|c|}
\hline miRNA ID & Sequence $\left(5^{\prime}-3^{\prime}\right)$ & Length(nt) & Start/end precursor & Length of precursors(nt) & Arm & MFE (kcal/mol) \\
\hline celery-miR-6 & ACGGAACCAGGATIITTGATGTCC & 24 & 95-195 & 101 & $5^{\prime}$ & -30.30 \\
\hline celery-miR-13 & ATTTGCTGTTAACGGGGTTCGAAC & 24 & 213-301 & 89 & $3^{\prime}$ & -27.90 \\
\hline celery-miR-18 & GTTAGTAGAAACAGATCCAAT & 21 & $392-489$ & 98 & $3^{\prime}$ & -45.20 \\
\hline celery-miR-22 & TAGGAAGACTGTTCTCAGTGA & 21 & $82-170$ & 89 & $5^{\prime}$ & -27.30 \\
\hline celery-miR-27 & TाTAGCTTGAACTATAGATT & 20 & $1522-1617$ & 96 & $3^{\prime}$ & -24.90 \\
\hline
\end{tabular}




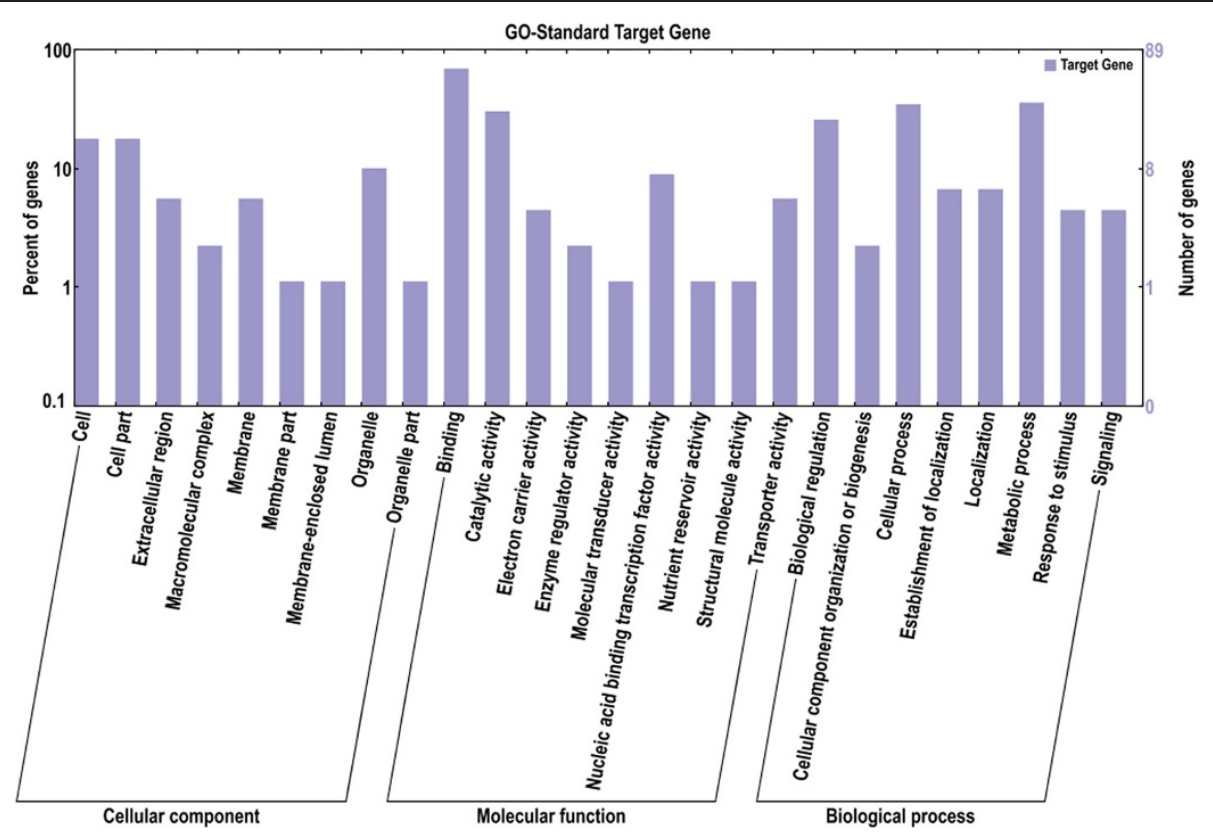

Figure $4 \mathrm{GO}$ categories and distribution of miRNA targets in celery.

Over six million reads of 16 to 30 nt were obtained from each library. Based on the sequence conservation of mature miRNAs, 418 and 341 conserved miRNAs were identified in 'Jinnan Shiqin' and 'Ventura' libraries, respectively. Most conserved miRNAs showed high sequence similarities to other plants, and were distributed in 37 conserved miRNA families. Axtell et al. [33] concluded that miRNA families were highly conserved in various plants, and that these miRNAs performed analogous regulatory functions. Some highly conserved miRNA families, including miR156,
miR159, miR166 and miR396, showed relatively high numbers of reads in the two celery varieties. Several studies have reported that these miRNAs have crucial roles in biotic and abiotic stress responses, plant development and fertility, and cell proliferation [14,34-36]. Other families, such as miR395 and miR812, had very low abundance in both celery varieties. These findings suggest that over long evolutionary timescales, the miRNAs evolved at different rates and have different roles in plant development. Putative targets gene were predicted to provide more information

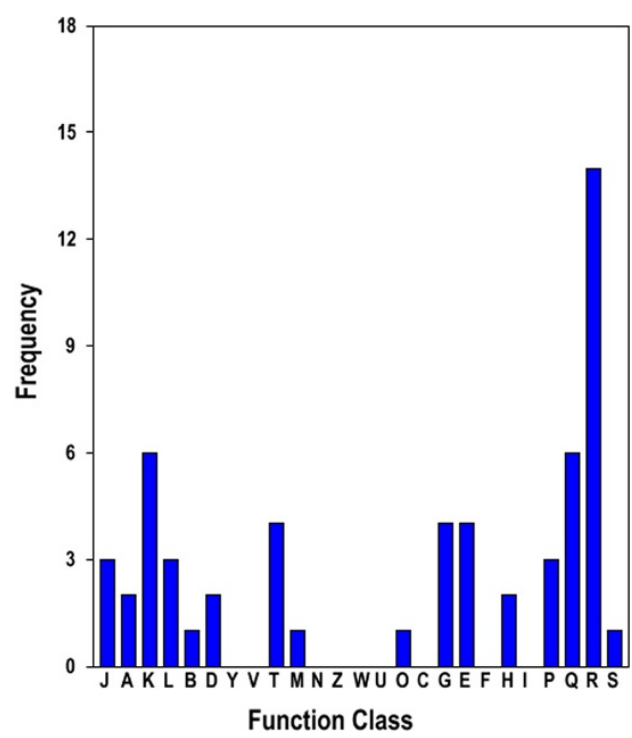

$\mathrm{J}$ : Translation, ribosomal structure and biogenesis

A: RNA processing and modification

K: Transcription

L: Replication, recombination and repair

B: Chromatin structure and dynamics

D: Cell cycle control, cell division, chromosome partitioning

Y: Nuclear structure

$\mathrm{V}$ : Defense mechanisms

T: Signal transduction mechanisms

M: Cell wall/membrane/envelope biogenesis

$\mathrm{N}$ : Cell motility

Z: Cytoskeleton

W: Extracellular structures

$\mathrm{U}$ : Intracellular trafficking, secretion, and vesicular transport

0 : Posttranslational modification, protein turnover, chaperones

C: Energy production and conversion

G: Carbohydrate transport and metabolism

E: Amino acid transport and metabolism

F: Nucleotide transport and metabolism

$\mathrm{H}$ : Coenzyme transport and metabolism

I: Lipid transport and metabolism

P: Inorganic ion transport and metabolism

Q: Secondary metabolites biosynthesis, transport and catabolism

$R$ : General function prediction only

S: Function unknown

Figure 5 COG function classification of consensus sequences in celery. 


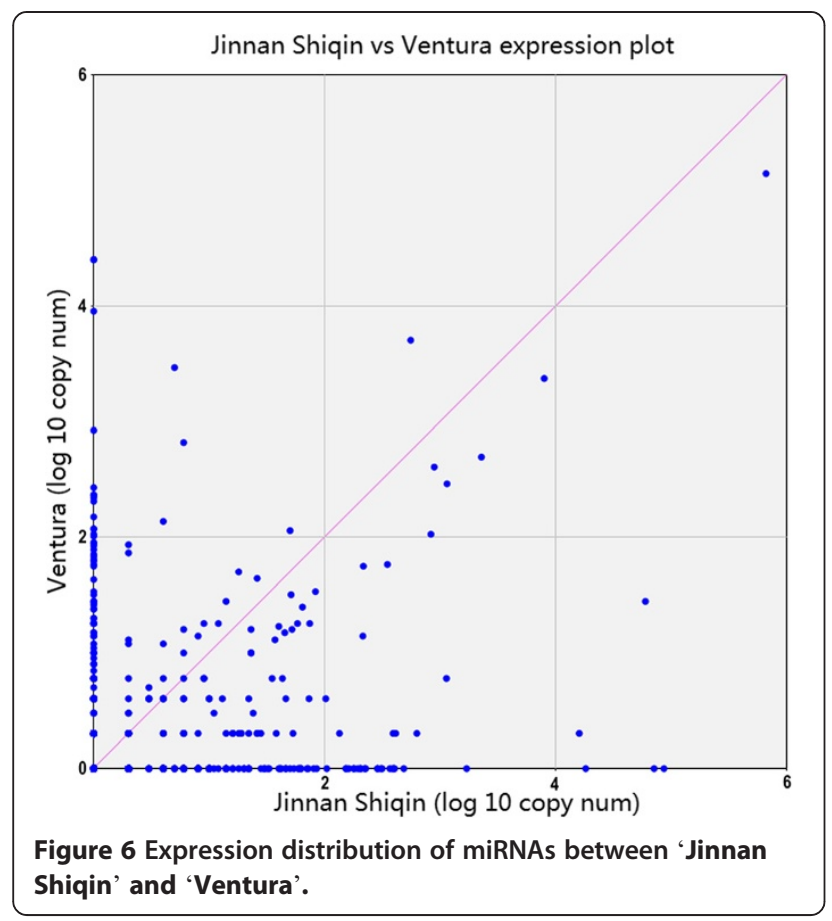

concerning the identified miRNAs, in which most target genes were involved in molecular functions and transcription pathways.

After searching for potential pre-miRNAs and predicting their hairpin-like structures, 13 and five novel miRNAs were identified in 'Jinnan Shiqin' and 'Ventura,' respectively. Surprisingly, among all the newly identified miRNAs, only one (celery-miR-27) was present in both varieties. Therefore, the functions of these novel celery miRNAs should be analyzed in future studies.

Significantly different abundance was seen for 221 miRNAs between 'Jinnan Shiqin' and 'Ventura'. Different miRNA genes accounted for a large proportion of all identified miRNAs. The two varieties have similar phenotypes, although they originate from different geographical areas: 'Jinnan Shiqin' from Asia and 'Ventura' from North America. This finding suggests that miRNA evolution in the same plant species is more conservative, and that variations in miRNA machinery in the two celery varieties may be critical for the differences in miRNA expression.

Considering the instability of the environment, plants are frequently challenged by various biotic and abiotic stresses, including temperature stress. Numerous studies have confirmed that small RNAs have important roles in biotic and abiotic stress responses in plants. Some miRNAs are temperature sensitive [37]. For instance, in wheat, miR393 and Ta-miR2002 were up-regulated after $0.5 \mathrm{~h}$ of heat treatment [38], while ten cold-regulated miRNAs were detected in Arabidopsis by microarray [39]. In castor bean, 41 miRNAs were down-regulated and four others were upregulated under cold stress compared with normal growth conditions [40]. In the present study, qRT-PCR method was used to investigate six miRNAs under cold and heat stress conditions. All miRNAs in both celery varieties showed sensitivity to temperature change, and abundance was remarkably up-regulated. This finding is consistent with results in Arabidopsis, poplar, and wheat [38,39,41]. The stronger response of miRNAs to cold compared to heat stress may be related to celery being a cool-season
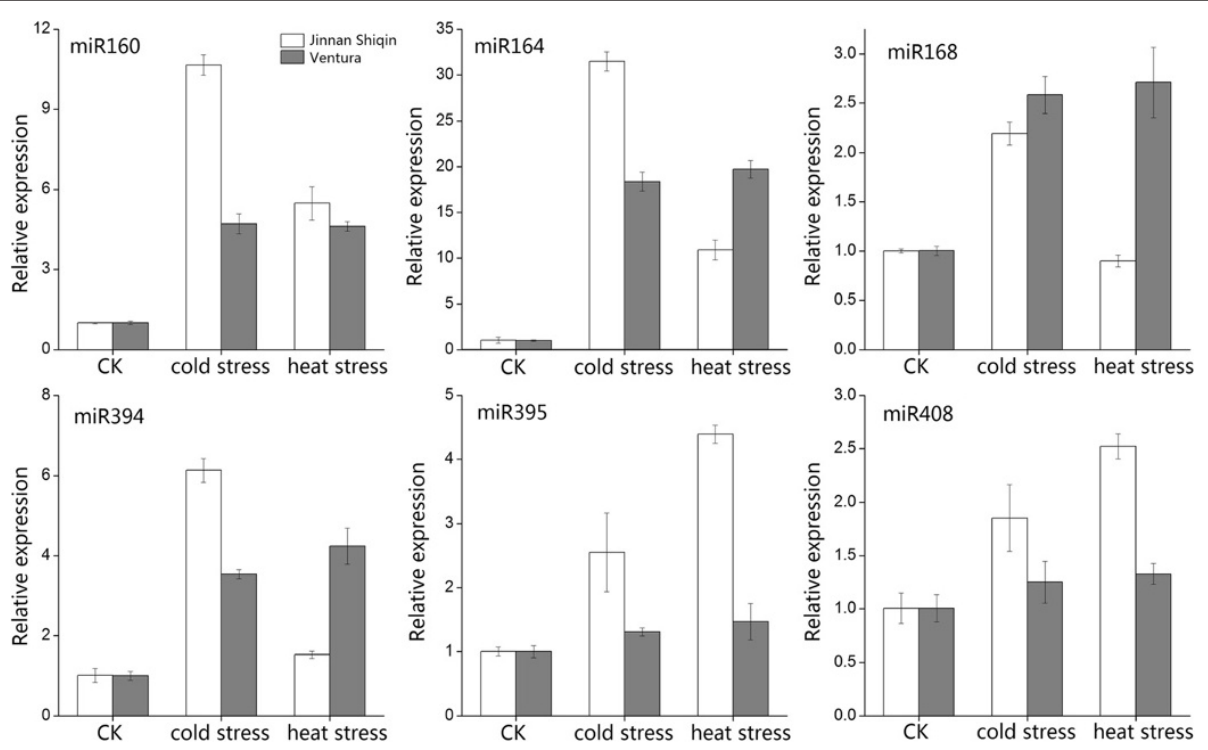

Figure 7 qRT-PCR analysis of miRNAs in 'Jinnan Shiqin' and 'Ventura'. CK: control check. Error bars were calculated based on three replicates. 
biennial plant. The abundance of most miRNAs was higher in 'Jinnan Shiqin' than in 'Ventura,', suggesting that 'Jinnan Shiqin' is more resistant to temperature stress. This result is consistent with the phenotypes of the two plants in relation to temperature stress. Thus, these miRNAs may have important roles in temperature stress defense. However, understanding the molecular mechanisms underlying these roles requires further study.

\section{Conclusion}

This is the first report to investigate small RNAs in celery; a large number of small RNAs were characterized as known and novel miRNAs. Putative target genes were predicted and then annotated by GO, COG, and KEGG databases to explore gene functions. The differential expression of miRNAs under temperature stress conditions between the two varieties suggests that the miRNA machinery in the two celery varieties may be different. Taken together, this study provides useful information for understanding the functions and regulatory mechanisms of miRNAs.

\section{Methods}

\section{Plant materials}

Celery plants (A. graveolens L. cvs. 'Jinnan Shiqin' and 'Ventura') were grown in pots containing soil:vermiculite mixture (3:1) in a controlled-environment growth chamber programmed for $16 \mathrm{~h} / 8 \mathrm{~h}$ at $25^{\circ} \mathrm{C} / 16^{\circ} \mathrm{C}$ for day/night and 3000 lux of light intensity. Two month-old plants were transferred to growth chambers set at $4^{\circ} \mathrm{C}$ or $38^{\circ} \mathrm{C}$ under the same light intensity and day length as above. Leaves and stems were collected $1 \mathrm{~h}$ after transfer to the hot or cold chambers, immediately frozen in liquid nitrogen, and then stored at $-80^{\circ} \mathrm{C}$ until use.

\section{RNA isolation, small RNA library development and Solexa sequencing}

Total RNA was extracted using an RNA kit (RNAsimple Total RNA Kit, Tiangen, Beijing, China) according to the manufacturer's instructions. RNA quantity and quality were examined using gel electrophoresis and a Nanodrop ND1000 spectrophotometer. Small RNAs of 18 to 30 nt were separated from total RNAs by polyacrylamide gel electrophoresis. Small RNA molecules were then ligated to Solexa adaptors at both 5'- and 3 '-ends, and then converted to cDNA by RT-PCR. The purified DNA samples were sequenced using an Illumina Cluster Station and Illumina Genome Analyzer. The data were submitted to the National Center for Biotechnology Information under accession number, SRR1125031.

\section{Prediction of conserved and novel miRNAs}

After removing the impure sequences (the low quality reads, adaptor reads, and reads with length $<16$ or length $>30$ ), unique reads were queried against ribosomal RNAs (rRNAs) and transfer RNAs (tRNAs) from GenBank (http://www.ncbi.nlm.nih.gov/). rRNA, tRNA, small nucleolar RNA (snoRNA), and small nuclear RNA (snRNA) sequences were obtained from Rfam (http:// rfam.sanger.ac.uk). Unique reads were also used for a nucleotide-nucleotide Basic Local Alignment Search Tool (BLASTn) search against the miRNA database (miRBase 16.0) to identify conserved miRNAs. To identify novel miRNAs, the Mireap program was used to obtain all candidate precursors with hairpin-like structures that were perfectly mapped by sequencing tags (http:// sourceforge.net/projects/mireap). The secondary structures of putative pre-miRNAs were checked using Mfold [42]. The criteria chosen for stem-loop hairpins were described by Meyers et al. [43].

\section{Prediction of potential miRNA target genes}

Target genes of miRNAs were predicted using the psRNA Target program (http://plantgrn.noble.org/psRNATarget). The rules used for target prediction were based on those suggested by Allen et al. [44] and Schwab et al. [45]. BLASTn hits with $<4$ mismatches were chosen as candidate targets, and then nucleotide 6-frame translationprotein (blastx) was used to obtain their putative functions.

\section{Functional annotation of the potential miRNA target genes}

To investigate the putative functions of potential target genes, the target sequences were annotated using diverse protein databases, including Gene Ontology (GO), Cluster of Orthologous Groups (COG) and Kyoto Encyclopedia of Genes and Genomes (KEGG) [30,31,46]. The GO categorization results are expressed as three independent hierarchies for biological processes, cellular components, and molecular functions.

\section{Differential expression of miRNAs between celery varieties}

To select differentially expressed miRNAs between the two libraries, the frequency of miRNAs was normalized to one million of the total number of miRNA reads in each sample. The selection method used was according to Audic and Claverie [47]. IDEG6 software [48] was used to analyze the differential expression.

\section{qRT-PCR analysis of miRNA abundance under} temperature stress conditions

Total RNA was extracted using an RNAsimple Total RNA Kit (Tiangen) according to the manufacturer's instructions. Small RNAs were reverse-transcribed into cDNA using the One Step PrimeScript ${ }^{\circ}$ miRNA cDNA Synthesis Kit (TaKaRa, Dalian, China). Quantitative realtime PCR (qRT-PCR) was performed using the MyiQ single-color real-time PCR detection system (Bio-Rad, 
Hercules, CA, USA). The reactions were carried out in a total volume of $20 \mu \mathrm{L}$ containing $2.0 \mu \mathrm{L}$ of diluted cDNA, $0.8 \mu \mathrm{L}$ of each primer, and $10 \mu \mathrm{L}$ of SYBR GreenI Mix with the following cycling profile: $95^{\circ} \mathrm{C}$ for $30 \mathrm{~s}$; followed by 40 cycles at $95^{\circ} \mathrm{C}$ for $5 \mathrm{~s}, 60^{\circ} \mathrm{C}$ for $20 \mathrm{~s}$; Melting curve analysis was performed ( 61 cycles at $65^{\circ} \mathrm{C}$ for $10 \mathrm{~s}$ ) to verify specific amplification. Each sample was processed in triplicate, and 5.8S rRNA was used as an internal control. The qRT-PCR primers are listed in Additional file 2: Table S2.

\section{Additional files}

Additional file 1: Table S1. The expression profiles of 221 differentially expressed miRNAs.

Additional file 2: Table S2. qRT-PCR primer sequences.

\section{Competing interests}

The authors declare that they have no competing interests.

\section{Authors' contributions}

ASX conceived and designed the experiments. MYL, FW, ZSX, QJ, GFT and JM performed the experiments. MYL, FW, JQ and ASX analyzed the data. ASX contributed reagents/materials/analysis tools. MYL wrote the paper. MYL and ASX revised the paper. All authors read and approved the final version of manuscript.

\section{Acknowledgements}

The research was supported by National Natural Science Foundation of China (31272175); New Century Excellent Talents in University (NCET-110670); Graduate Educated Innovation Project of Jiangsu (CXZZ13_0297); Jiangsu Natural Science Foundation (BK20130027); Priority Academic Program Development of Jiangsu Higher Education Institutions (PAPD) and Jiangsu Shuangchuang Project.

Received: 30 June 2013 Accepted: 24 March 2014

Published: 27 March 2014

\section{References}

1. Obernosterer G, Leuschner PJ, Alenius M, Martinez J: Post-transcriptional regulation of microRNA expression. RNA (New York, NY) 2006, 12(7):1161-1167.

2. Kren BT, Wong PY, Sarver A, Zhang X, Zeng Y, Steer CJ: MicroRNAs identified in highly purified liver-derived mitochondria may play a role in apoptosis. RNA Biol 2009, 6(1):65-72.

3. Lee CT, Risom T, Strauss WM: Evolutionary conservation of microRNA regulatory circuits: an examination of microRNA gene complexity and conserved microRNA-target interactions through metazoan phylogeny. DNA Cell Biol 2007, 26(4):209-218.

4. Voinnet O: Origin, biogenesis, and activity of plant MicroRNAs. Cell 2009, 136(4):669-687.

5. Lee RC, Feinbaum RL, Ambros V: The C. elegans heterochronic gene lin-4 encodes small RNAs with antisense complementarity to lin-14. Cell 1993, 75(5):843-854

6. Reinhart BJ, Slack FJ, Basson M, Pasquinelli AE, Bettinger JC, Rougvie AE, Horvitz HR, Ruvkun G: The 21-nucleotide let-7 RNA regulates developmental timing in Caenorhabditis elegans. Nature 2000, 403(6772):901-906.

7. Brennecke J, Hipfner DR, Stark A, Russell RB, Cohen SM: bantam encodes a developmentally regulated microRNA that controls cell proliferation and regulates the proapoptotic gene hid in Drosophila. Cell 2003, 113(1):25-36.

8. De Lima JC, Loss-Morais G, Margis R: MicroRNAs play critical roles during plant development and in response to abiotic stresses. Genet Mol Biol 2012, 35(4 (suppl)):1069-1077.
9. Chen L, Ren $Y$, Zhang Y, Xu J, Zhang Z, Wang Y: Genome-wide profiling of novel and conserved Populus microRNAs involved in pathogen stress response by deep sequencing. Planta 2012, 235(5):873-883.

10. Huntzinger $E$, Izaurralde $E$ : Gene silencing by microRNAs: contributions of translational repression and mRNA decay. Nat Rev Genet 2011, 12(2):99-110.

11. Chen J, Li WX, Xie D, Peng JR, Ding SW: Viral virulence protein suppresses RNA silencing-mediated defense but upregulates the role of microrna in host gene expression. Plant Cell 2004, 16(5):1302-1313.

12. Bazzini AA, Hopp HE, Beachy RN, Asurmendi S: Infection and coaccumulation of tobacco mosaic virus proteins alter microRNA levels, correlating with symptom and plant development. Proc Natl Acad Sci U S A 2007, 104(29):12157-12162.

13. Aukerman MJ, Sakai $\mathrm{H}$ : Regulation of flowering time and floral organ identity by a MicroRNA and its APETALA2-like target genes. Plant Cell 2003, 15(11):2730-2741.

14. Li X, Bian H, Song D, Ma S, Han N, Wang J, Zhu M: Flowering time control in ornamental gloxinia (Sinningia speciosa) by manipulation of miR159 expression. Annals of botany 2013, 111(5):791-799.

15. Mallory AC, Bartel DP, Bartel B: MicroRNA-directed regulation of Arabidopsis AUXIN RESPONSE FACTOR17 is essential for proper development and modulates expression of early auxin response genes. The Plant cell 2005, 17(5):1360-1375.

16. Sunkar R, Zhu JK: Novel and stress-regulated microRNAs and other small RNAs from Arabidopsis. Plant Cell 2004, 16(8):2001-2019.

17. Raffo A, Sinesio F, Moneta E, Nardo N, Peparaio M, Paoletti F: Internal quality of fresh and cold stored celery petioles described by sensory profile, chemical and instrumental measurements. Eur Food Res Technol 2005, 222(5-6):590-599.

18. Mongeau R, Siddiqui IR, Emery J, Brassard R: Effect of dietary fiber concentrated from celery, parsnip, and rutabaga on intestinal function, serum cholesterol, and blood glucose response in rats. J Agric Food Chem 1990, 38(1):195-200.

19. Song $X$, Wang $Y$ : Research progress in the medicinal function of celery. J Anhui Agric Sci 2008, 36(15):6360-6361.

20. Eriksson NE, Moller C, Werner S, Magnusson J, Bengtsson U, Zolubas M: Self-reported food hypersensitivity in Sweden, Denmark, Estonia, Lithuania, and Russia. J Investig Allergol Clin Immunol 2004, 14(1):70-79.

21. Hoffmann-Sommergruber K, Demoly P, Crameri R, Breiteneder H, Ebner C, Laimer Da Camara Machado M, Blaser K, Ismail C, Scheiner O, Bousquet J, Menz G: IgE reactivity to Api g 1, a major celery allergen, in a Central European population is based on primary sensitization by Bet $\mathrm{v} 1$. J Allergy Clin Immunol 1999, 104(2 Pt 1):478-484.

22. Bi Y, Liu G, Yang R: MicroRNAs: novel regulators during the immune response. J Cell Physiol 2009, 218(3):467-472.

23. Rajagopalan R, Vaucheret H, Trejo J, Bartel DP: A diverse and evolutionarily fluid set of microRNAs in Arabidopsis thaliana. Genes Dev 2006, 20(24):3407-3425.

24. Song C, Wang C, Zhang C, Korir NK, Yu H, Ma Z, Fang J: Deep sequencing discovery of novel and conserved microRNAs in trifoliate orange (Citrus trifoliata). BMC Genomics 2010, 11:431.

25. Chi X, Yang Q, Chen X, Wang J, Pan L, Chen M, Yang Z, He Y, Liang X, Yu S: Identification and characterization of microRNAs from peanut (Arachis hypogaea L.) by high-throughput sequencing. PloS One 2011, 6(11):e27530.

26. Donaire L, Pedrola L, Rosa Rde L, Llave C: High-throughput sequencing of RNA silencing-associated small RNAs in olive (Olea europaea L.). PloS One 2011, 6(11):e27916.

27. Pontes O, Costa-Nunes P, Vithayathil P, Pikaard CS: RNA polymerase V functions in Arabidopsis interphase heterochromatin organization independently of the 24-nt siRNA-directed DNA methylation pathway. Mol Plant 2009, 2(4):700-710.

28. Jia Y, Lisch DR, Ohtsu K, Scanlon MJ, Nettleton D, Schnable PS: Loss of RNA-dependent RNA polymerase 2 (RDR2) function causes widespread and unexpected changes in the expression of transposons, genes, and 24-nt small RNAs. PLoS Genet 2009, 5(11):e1000737.

29. Bonnet E, Wuyts J, Rouze P, Van de Peer Y: Evidence that microRNA precursors, unlike other non-coding RNAs, have lower folding free energies than random sequences. Bioinformatics 2004, 20(17):2911-2917.

30. Ashburner M, Ball CA, Blake JA, Botstein D, Butler H, Cherry JM, Davis AP, Dolinski K, Dwight SS, Eppig JT, Harris MA, Hill DP, Issel-Tarver L, Kasarskis A, Lewis S, Matese JC, Richardson JE, Ringwald M, Rubin GM, Sherlock G: 
Gene ontology: tool for the unification of biology. The Gene Ontology Consortium. Nat Genet 2000, 25(1):25-29.

31. Tatusov RL, Fedorova ND, Jackson JD, Jacobs AR, Kiryutin B, Koonin EV Krylov DM, Mazumder R, Mekhedov SL, Nikolskaya AN, Rao BS, Smirnov S, Sverdlov AV, Vasudevan S, Wolf YI, Yin JJ, Natale DA: The COG database: an updated version includes eukaryotes. BMC Bioinforma 2003, 4:41.

32. Fu N, Wang $Q$, Shen HL: De novo assembly, gene annotation and marker development using illumina paired-End transcriptome sequences in celery (apium graveolens L.). PloS One 2013, 8(2):e57686.

33. Axtell MJ, Snyder JA, Bartel DP: Common functions for diverse small RNAs of land plants. Plant Cell 2007, 19(6):1750-1769.

34. Khraiwesh B, Zhu JK, Zhu J: Role of miRNAs and siRNAs in biotic and abiotic stress responses of plants. Biochim Biophys Acta 2012, 1819(2):137-148.

35. Li Y, Li C, Ding G, Jin Y: Evolution of MIR159/319 microRNA genes and their post-transcriptional regulatory link to siRNA pathways. BMC Evol Biol 2011, 11(1):122.

36. Rodriguez RE, Mecchia MA, Debernardi JM, Schommer C, Weigel D, Palatnik JF: Control of cell proliferation in Arabidopsis thaliana by microRNA miR396. Development 2010, 137(1):103-112.

37. Zhou X, Wang G, Sutoh K, Zhu JK, Zhang W: Identification of coldinducible microRNAs in plants by transcriptome analysis. Biochim Biophys Acta 2008, 1779(11):780-788

38. Xin M, Wang Y, Yao Y, Xie C, Peng H, Ni Z, Sun Q: Diverse set of microRNAs are responsive to powdery mildew infection and heat stress in wheat (Triticum aestivum L.). BMC Plant Biol 2010, 10:123.

39. Liu HH, Tian X, Li YJ, Wu CA, Zheng CC: Microarray-based analysis of stress-regulated microRNAs in Arabidopsis thaliana. RNA (New York, NY) 2008, 14(5):836-843.

40. Zeng C, Wang W, Zheng Y, Chen X, Bo W, Song S, Zhang W, Peng M: Conservation and divergence of microRNAs and their functions in Euphorbiaceous plants. Nucleic Acids Res 2010, 38(3):981-995.

41. Lu S, Sun YH, Chiang VL: Stress-responsive microRNAs in Populus. Plant J 2008, 55(1):131-151.

42. Zuker M: Mfold web server for nucleic acid folding and hybridization prediction. Nucleic Acids Res 2003, 31(13):3406-3415.

43. Meyers BC, Axtell MJ, Bartel B, Bartel DP, Baulcombe D, Bowman JL, Cao X, Carrington JC, Chen X, Green PJ, Griffiths-Jones S, Jacobsen SE, Mallory AC, Martienssen RA, Poethig RS, Qi Y, Vaucheret H, Voinnet O, Watanabe Y, Weigel D, Zhu JK: Criteria for annotation of plant MicroRNAs. Plant Cell 2008, 20(12):3186-3190.

44. Allen $\mathrm{E}$, Xie Z, Gustafson AM, Carrington JC: microRNA-directed phasing during trans-acting siRNA biogenesis in plants. Cell 2005, 121(2):207-221.

45. Schwab R, Palatnik JF, Riester M, Schommer C, Schmid M, Weigel D: Specific effects of MicroRNAs on the plant transcriptome. Dev Cell 2005, 8(4):517-527.

46. Kanehisa M, Goto S: KEGG: kyoto encyclopedia of genes and genomes. Nucleic Acids Res 2000, 28(1):27-30.

47. Audic $\mathrm{S}$, Claverie JM: The significance of digital gene expression profiles. Genome Res 1997, 7(10):986-995.

48. Romualdi C, Bortoluzzi S, d'Alessi F, Danieli GA: IDEG6: a web tool for detection of differentially expressed genes in multiple tag sampling experiments. Physiol Genomics 2003, 12(2):159-162.

doi:10.1186/1471-2164-15-242

Cite this article as: Li et al:: High throughput sequencing of two celery varieties small RNAs identifies microRNAs involved in temperature stress response. BMC Genomics 2014 15:242

\section{Submit your next manuscript to BioMed Central and take full advantage of:}

- Convenient online submission

- Thorough peer review

- No space constraints or color figure charges

- Immediate publication on acceptance

- Inclusion in PubMed, CAS, Scopus and Google Scholar

- Research which is freely available for redistribution 